\title{
GLOBAL EXISTENCE FOR THE NONLINEAR FRACTIONAL SCHRÖDINGER EQUATION WITH FRACTIONAL DISSIPATION.
}

\author{
MOHAMAD DARWICH.
}

Wednesday $28^{\text {th }}$ February, 2018

\begin{abstract}
We consider the initial value problem for the fractional nonlinear Schrödinger equation with a fractional dissipation. Global existence and scattering are proved depending on the order of the fractional dissipation.
\end{abstract}

\section{INTRODUCTION}

Consider the Cauchy problem for the damped fractional nonlinear Schrödinger equation

$$
\left\{\begin{array}{l}
i u_{t}-(-\Delta)^{\alpha} u+|u|^{p-1} u+i a(-\Delta)^{s} u=0,(t, x) \in\left[0, \infty\left[\times \mathbb{R}^{d} .\right.\right. \\
u(0)=u_{0}
\end{array}\right.
$$

where $a>0$ is the coefficient of friction, $d \geq 2, \alpha \in\left(\frac{d}{2 d-1}, 1\right), s>0$ with $L^{2}$-critical nonlinearity i.e $p=1+\frac{4 \alpha}{d}$.

In the classical case $(\alpha=1$ and $a=0)$ equation (1.1) arises in various areas of nonlinear optics, plasma physics and fluid mechanics to describe propagation phenomena in dispersive media.

When $a=0$ and $0<\alpha<1$ equation (1.1) (called FNLS : Fractional NLS) can be seen as a canonical model for a nonlocal dispersive PDE with focusing nonlinearity that can exhibit solitary waves, turbulence phenomena which has been studied by many authors [20, 8, [10, [11, [21, 23] and 29] in mathematics, numerics, and physics. The FNLS equation is a fundamental equation of fractional quantum mechanics, which was derived by Laskin [24, 25] as a result of extending the Feynman path integral, from the Brownian-like to Levy-like quantum mechanical paths. The Cauchy problem for FNLS was studied in [14] and [15] and proved that it is wellposed and scatters in the radial energy space and in [16] the author proves that the equation is globaly well posed for small data.

In this this paper we complete the $L^{2}$-critical FNLS equation with a fractional laplacian of order $2 s, s>0$. The fractional laplacian is commonly used to model fractal (anomalous) diffusion related to the Lévy flights (see

Key words and phrases. Damped Fractional Nonlinear Schrödinger Equation, Global existence. 
e.g. Stroock [27, Bardos and all 3], Hanyga [18). It also appears in the physical literature to model attenuation phenomena of acoustic waves in irregular porous random media (cf. Blackstock [4], Gaul [13], Chen-Holm [9]).

Note that for $s=0$, the global existence for (1.1) was proved in [28] for a large daming tem ( i.e for large $a>0$ ), in this paper we will obtain the global existence result for any damping term $a>0$.

Finally, the case $\alpha=1$ and a nonlinear damping of the type $i a|u|^{p} u$, has been studied by Antonelli-Sparber and Antonelli-Carles-Sparber (cf. [1] and [2]). In this case the origin of the nonlinear damping term is multiphoton absorption.

The purpose of this paper is to prove some global well-posedness and scattering results for (1.1) in the radial case and the rest of the paper is organized as follows. Section 2 is devoted to prove the local existence results. In section 3 we will show the main results i.e the global well-posedness of equation (1.1) and the scattering.

Now let us define the following quantities:

$L^{2}$-norm : $m(u)=\|u\|_{L^{2}}=\left(\int|u(x)|^{2} d x\right)^{1 / 2}$.

Energy : $E(u)=\frac{1}{2}\left\|(-\Delta)^{\frac{\alpha}{2}} u\right\|_{L^{2}}^{2}-\frac{d}{4 \alpha+2 d}\|u\|_{L^{\frac{4 \alpha}{d}+2}}^{\frac{4 \alpha}{d}+2}$.

However, it is easy to prove that if $u$ is a smooth solution of (1.1) on $[0, T[$, then for all $t \in[0, T[$ it holds

$$
\begin{gathered}
\frac{d}{d t}(m(u(t)))=-a\left\|(-\Delta)^{\frac{s}{2}} u\right\|_{L^{2}}^{2} ; \\
\frac{d}{d t}(E(u(t)))=-a \int\left|(-\Delta)^{\frac{s+\alpha}{2}} u(t)\right|^{2}+a \Im \int\left((-\Delta)^{s} u(t)\right)|u(t)|^{p-1} \bar{u}(t) .
\end{gathered}
$$

Let us now state our results:

Theorem 1.1. Let $d \geq 2, \alpha \in\left(\frac{d}{2 d-1}, 1\right)$ and $0<s<\alpha$, such that $s+\alpha \geq 1$ then there exists a real number $\beta>0$ such that for any initial datum $u_{0} \in$ $H_{r d}^{\alpha}\left(\mathbb{R}^{d}\right)$ with $\left\|u_{0}\right\|_{L^{2}}<\beta$, the emanating solution $u$ is global in $H_{r d}^{\alpha}\left(\mathbb{R}^{d}\right)$.

Theorem 1.2. Let $d \geq 2, \alpha \in\left(\frac{d}{2 d-1}, 1\right)$ and $s \geq \alpha$. Then the Cauchy problem (1.1) is globally well-posed in $H_{r d}^{\alpha}\left(\mathbb{R}^{d}\right)$.

Theorem 1.3. Let $\alpha \in\left(\frac{d}{2 d-1}, 1\right), s=\alpha, u_{0} \in H_{r d}^{\alpha}\left(\mathbb{R}^{d}\right)$ and $u \in C\left(\mathbb{R}^{+}, H_{r d}^{\alpha}\right)$ be the global solution to (1.1). Then:

(1) There exists $u_{+} \in L^{2}$ such that $\left\|\left(u-S_{a, \alpha, s}(.) u_{+}\right)(t)\right\|_{L^{2}} \longrightarrow 0$, as $t \longrightarrow+\infty$.

(2) $\|u\|_{L^{\frac{4 \alpha}{d}+2}\left(\mathbb{R}_{+}^{*} \times \mathbb{R}^{d}\right)} \longrightarrow 0$, when $a \longrightarrow+\infty$.

Acknowledgments : The author thanks Luc Molinet for his valuable remarks and comments in this paper.

\section{LOCAL EXISTENCE RESUlt}

Recall that the main tools to prove the local existence results for the FNLS equation are the Strichartz estimates for the associated linear propagator 
$e^{i(-\Delta)^{\alpha} t}$. Let us mention that in the case $a>0$ the same results on the local Cauchy problem for (1.1) can be established in exactly the same way as in the case $a=0$, since the same Strichartz estimates hold.

\subsection{Strichartz estimate.}

Definition 2.1. A pair $(q, r), q, r \geq 2$ is said to be admissible if:

$$
\frac{4 d+2}{2 d-1} \leq q \leq \infty, \frac{2}{q}+\frac{2 d-1}{r} \leq d-\frac{1}{2},
$$

or

$$
2 \leq \frac{4 d+2}{2 d-1}, \frac{2}{q}+\frac{2 d-1}{r}<d-\frac{1}{2} .
$$

These Strichartz estimates read in the following proposition see [17]:

Proposition 2.1. Suppose $d \geq 2, a=0$ and $u$ be a radial solution of 1.1, then for every admissible pair $(q, r)$ satisfie the following condition:

$$
\frac{2}{q}+\frac{d}{r}=\frac{d}{2}-\gamma, \frac{2}{\tilde{q}}+\frac{d}{\tilde{r}}=\frac{d}{2}+\gamma
$$

it holds:

$$
\|u\|_{\left(L_{t}^{q} L_{x}^{r} \cap L_{t}^{\infty} H^{\gamma}\right)} \leq\left\|u_{0}\right\|_{\left(L_{t}^{q} L_{x}^{r} \cap L_{t}^{\infty} H^{\gamma}\right)}+\left\||u|^{p}\right\|_{\left(L^{\tilde{q}^{\prime}} L^{\left.\tilde{r}^{\prime}\right)}\right.}
$$

For $a \geq 0, \alpha \geq 0$ and $s \geq 0$ we denote by $S_{a, \alpha, s}$ the linear semi-group associated with (1.1), i.e. $S_{a, \alpha, s}(t)=e^{i(-\Delta)^{\alpha} t-a(-\Delta)^{s} t}$. It is worth noticing that $S_{a, \alpha, s}$ is irreversible.

We will see in the following proposition that that the linear semi-group $S_{a, \alpha, s}$ enjoys the same Strichartz estimates as $e^{i(-\Delta)^{\alpha} t}$.

Proposition 2.2. Let $d \geq 2, u_{0} \in H^{\gamma}\left(\mathbb{R}^{d}\right), \gamma \in \mathbb{R}, s \geq 0$ and $\frac{d}{2 d-1}<\alpha<1$. Then for every admissible pair $(q, r)$ satisfie the following condition:

$$
\frac{2}{q}+\frac{d}{r}=\frac{d}{2}-\gamma, \frac{2}{\tilde{q}}+\frac{d}{\tilde{r}}=\frac{d}{2}+\gamma
$$

it holds:

$$
\|u\|_{\left(L_{t}^{q} L_{x}^{r} \cap L_{t}^{\infty} H^{\gamma}\right)} \leq\left\|u_{0}\right\|_{\left(L_{t}^{q} L_{x}^{r} \cap L_{t}^{\infty} H^{\gamma}\right)}+\left\||u|^{p}\right\|_{\left(L^{\tilde{q}^{\prime}} L^{\left.\tilde{r}^{\prime}\right)}\right.}
$$

Proof. Let for any $t \geq 0, H_{a, s}(t, x)=\int e^{-i x \xi} e^{-a t|\xi|^{2 s}} d \xi$, it holds

$$
S_{a, \alpha, s}(t) \varphi=H_{a, s}(t, \cdot) * e^{i t(-\Delta)^{\alpha}} \varphi, \quad \forall t \geq 0 .
$$

Noticing that for $s>0,\left\|H_{a, s}(t, .)\right\|_{L^{1}}=\left\|H_{1, s}(1, .)\right\|_{L^{1}}$ and that, according to Lemma 2.1 in [26], $H_{1, s}(1,.) \in L^{1}\left(\mathbb{R}^{d}\right)$ for $s>0$. Now let $g=|u|^{p}$ we 
have that:

$$
\begin{aligned}
\|u\|_{\left(L_{t}^{q} L_{x}^{r} \cap L_{t}^{\infty} H^{\gamma}\right)} & =\left\|S_{a, \alpha, s}(t)\left(u_{0}\right)-i \int_{0}^{t} S_{a, \alpha, s}\left(t-t^{\prime}\right) f\left(t^{\prime}\right) d t^{\prime}\right\|_{\left(L_{t}^{q} L_{x}^{r} \cap L_{t}^{\infty} H^{\gamma}\right)} \\
& \leq\left\|S_{a, \alpha, s}(t)\left(u_{0}\right)\right\|_{\left(L_{t}^{q} L_{x}^{r} \cap L_{t}^{\infty} H^{\gamma}\right)}+\left\|\int_{0}^{t} S_{a, \alpha, s}\left(t-t^{\prime}\right) f\left(t^{\prime}\right)\right\|_{\left(L_{t}^{q} L_{x}^{r} \cap L_{t}^{\infty} H^{\gamma}\right)} \\
& =\left\|H_{a, s}(t,) * e^{i t(-\Delta)^{\alpha}}\left(u_{0}\right)\right\|_{\left(L_{t}^{q} L_{x}^{r} \cap L_{t}^{\infty} H^{\gamma}\right)} \\
& +\left\|\int_{0}^{t} H_{a, s}\left(t-t^{\prime}\right) * e^{i t(-\Delta)^{\alpha}}\left(f\left(t^{\prime}\right)\right) d t^{\prime}\right\|_{\left(L_{t}^{q} L_{x}^{r} \cap L_{t}^{\infty} H^{\gamma}\right)} \\
& \leq\left\|H_{a, s}(t,)\right\|_{L^{1}}\left\|e^{i t(-\Delta)^{\alpha}}\left(u_{0}\right)\right\|_{\left(L_{t}^{q} L_{x}^{r} \cap L_{t}^{\infty} H^{\gamma}\right)} \\
& +\left\|H_{a, s}\left(t-t^{\prime},\right)\right\|_{L^{1}}\left\|\int_{0}^{t} H_{a, s}\left(t-t^{\prime}\right) * e^{i t(-\Delta)^{\alpha}}\left(f\left(t^{\prime}\right)\right) d t^{\prime}\right\|_{\left(L_{t}^{q} L_{x}^{r} \cap L_{t}^{\infty} H^{\gamma}\right)} \\
& \leq\left\|u_{0}\right\|_{H^{\gamma}}+\|f\|_{L^{\tilde{q}^{\prime}} L^{\tilde{r}^{\prime}}}
\end{aligned}
$$

With Proposition 2.2 in the hand, it is not too hard to check that the local existence results for equation 1.1 (see Theorem 4.2 in [17]). More precisely, we have the following statement:

Proposition 2.3. Let $a \geq 0, \alpha \in\left(\frac{2 d}{2 d-1}, 1\right), s>0$ and $u_{0} \in H_{r d}^{\alpha}\left(\mathbb{R}^{d}\right)$ with $d \geq 2$. There exists $T>0$ and a unique solution $u \in C\left([0, T] ; H_{r d}^{\alpha}\right) \cap$ $L_{T}^{\frac{4 \alpha}{d}+2} L^{\frac{4 \alpha}{d}+2}$ to (1.1) emanating from $u_{0}$.

\section{GLOBAL EXISTENCE RESULTS}

In this section, we will prove the global existence results. Let us start by the second theorem:

3.1. Proof of theorem 1.2. To prove theorem 1.2, we will establish an a priori estimate on the Strichartz norm.

Proposition 3.1. Suppose that $a>0, \alpha>0$ and $s>0$. Then there exists $\epsilon>0$ such that if $u_{0}$ in $H_{r d}^{\alpha}\left(\mathbb{R}^{d}\right)$ and $\left\|S_{a, \alpha, s}(\cdot) u_{0}\right\|_{L^{\frac{4 \alpha}{d}+2} L^{\frac{4 \alpha}{d}+2}} \leq \epsilon$, then the maximal time of the existence $T^{*}$ of the solution emanating from $u_{0}$ equal to $+\infty$.

To prove this claim, we will use the following proposition:

Proposition 3.2. There exists $\delta>0$ with the following property. If $u_{0} \in$ $L^{2}\left(R^{d}\right)$ and $T \in(0, \infty]$ are such that $\left\|S_{a, \alpha, s}(.) u_{0}\right\|_{L^{p+1}\left([0, T], L^{p+1}\right)}<\delta$, there exists a unique solution $u \in C\left([0, T], L^{2}\left(R^{d}\right)\right) \cap L^{p+1}\left([0, T], L^{p+1}\left(R^{d}\right)\right)$ of (1.1). In addition, $u \in L^{q}\left([0, T], L^{r}\left(R^{d}\right)\right)$ for every admissible pair $(q, r)$, for $t \in[0, T]$. Finally, $u$ depends continuously in $C\left([0, T], L^{2}\left(R^{d}\right)\right) \cap L^{p+1}\left([0, T], L^{p+1}\left(R^{d}\right)\right)$ on $u_{0} \in L^{2}\left(R^{d}\right)$. If $u_{0} \in H^{\alpha}\left(R^{d}\right)$, then $u \in C\left([0, T], H^{\alpha}\left(R^{d}\right)\right)$.

See [6] for the proof

Proposition 3.3. Let $u_{0} \in H^{\alpha}$ and $u$ be the solution of 1.1. Let $T^{*}$ be the maximal time of the existence of $u$ such that $\|u\|_{L^{\frac{4 \alpha}{d}+2}\left(\left[0, T^{*}[) L^{\frac{4 \alpha}{d}+2}\right.\right.}<+\infty$, then $T^{*}=+\infty$. 
Proof. Observe that $\left\|S_{a, \alpha, s}(.) u_{0}\right\|_{L^{\frac{4 \alpha}{d}+2}\left[0, T\left[L^{\frac{4 \alpha}{d}+2}\right.\right.} \longrightarrow 0$ as $T \longrightarrow 0$. Thus for sufficiently small $T$, the hypotheses of Proposition 3.2 are satisfied. Applying iteratively this proposition, we can construct the maximal solution $\left.u \in C\left(\left[0, T^{*}\right), H^{\alpha}\left(\mathbb{R}^{d}\right)\right)\right) \cap L^{\frac{4 \alpha}{d}+2}\left(\left[0, T^{*}\right), L^{\frac{4 \alpha}{d}+2}\left(\mathbb{R}^{d}\right)\right)$ of (1.1). We proceed by contradiction, assuming that $T^{*}<\infty$, and $\|u\|_{L^{\frac{4 \alpha}{d}+2}(] 0, T\left[, L^{\frac{4 \alpha}{d}+2}\right)}<\infty$.

Let $t \in\left[0, T^{*}\right)$. For every $s \in\left[0, T^{*}-t\right)$ we have

$$
S(.) u(t)=u(t+.)+i \int_{0}^{t} S_{a, \alpha, s}(t-\tau)\left(|u|^{p-1} u\right) d \tau .
$$

Then by Strichartz estimate, there exists $K>0$ such that:

$\left\|S_{a, \alpha, s}(.) u(t)\right\|_{L^{\frac{4 \alpha}{d}+2}\left(\left[0, T^{*}-t\right), L^{\frac{4 \alpha}{d}+2}\left(\mathbb{R}^{d}\right)\right)} \leq\|u\|_{L^{\frac{4 \alpha}{d}+2}(] t, T^{*}\left[, L^{\frac{4 \alpha}{d}}+2\right)}+K\left(\|u\|_{L^{\frac{4 \alpha}{d}+2}(] t, T^{*}\left[, L^{\frac{4 \alpha}{d}+2}\right)}\right)^{\frac{4}{d}+1}$

Therefore, for $t$ fixed close enough to $T^{*}$, it follows that

$$
\left\|S_{a, s, \alpha}(.) u(t)\right\|_{L^{\sigma}\left(\left[0, T^{*}-t\right), L^{\sigma}\left(\mathbb{R}^{d}\right)\right)} \leq \delta .
$$

Applying Proposition 3.2. we find that $u$ can be extended after $T^{*}$, which contradicts the maximality.

Now let us return to the proof of proposition 3.1 .

Let $q=\frac{4 \alpha(1+p)}{d(p-1)}=\frac{4 \alpha}{d}+2$ and $q^{\prime}=\frac{4 \alpha+d}{4 \alpha+2 d}$, then $q^{\prime}$ verifies: $\frac{1}{q^{\prime}}=\frac{1}{q}+\frac{p-1}{\theta}$, by Holder inequalities and the Strichartz estimate we obtain:

$$
\|u\|_{L_{T}^{q} L^{p+1}} \lesssim\left\|u_{0}\right\|_{L^{2}}+\left\|u|u|^{p-1}\right\|_{L^{q^{\prime}} L^{\frac{p+1}{p}}} \lesssim\left\|u_{0}\right\|_{L^{2}}+\|u\|_{L^{\theta} L^{p+1}}^{p}\|u\|_{L^{q} L^{p+1}}
$$

Remark that :

$$
\frac{2 \alpha}{\theta}=d\left(\frac{1}{2}-\frac{1}{r}\right), \frac{2 \alpha}{\theta}=d\left(\frac{1}{2}-\frac{1}{r}\right)
$$

Now $\frac{2}{\theta}+\frac{2 d-1}{r}<d-\frac{1}{2}$ iff $\alpha>\frac{d}{2 d-1}$

If we take $\gamma=0$ in the Strichartz estimate this gives with Holder inequality:

$$
\begin{aligned}
\|u\|_{L^{\theta} L^{r}} & \lesssim\left\|S_{a, \alpha, s}() u_{0}\right\|_{L^{\theta} L^{r}}+\left\||u|^{p}\right\|_{L^{\tilde{\theta}} L^{\tilde{r}}} \\
& \lesssim \epsilon+\|u\|_{L^{\theta} L^{r}}^{p} \\
& \lesssim \epsilon
\end{aligned}
$$

Noticing that the fractional Leibniz rule (see [22]) and by Holder inequality, leads to

$$
\begin{aligned}
\left\|\left.u\right|^{p-1} u\right\|_{L^{q^{\prime} H^{\alpha, r^{\prime}}}} & \lesssim\left\|(-\Delta)^{\alpha} u\right\|_{L^{q} L^{r}}\|u\|_{L^{\theta} L^{r}}^{p-1}+\|u\|_{L^{\theta} L^{r}}^{p-1}\|u\|_{L^{q} L^{r}} \\
& \lesssim\|u\|_{L^{\theta} L^{r}}^{p-1}\|u\|_{L^{q} H^{\alpha, r}} \\
& \lesssim \epsilon^{p-1}\|u\|_{L^{q} H^{\alpha, r}}
\end{aligned}
$$

This implies, with Strichartz estimates in the hand, that:

$$
\|u\|_{L^{q} H^{\alpha, r}} \lesssim\left\|u_{0}\right\|_{H^{\alpha}}+\epsilon^{p-1}\|u\|_{L^{q} H^{\alpha, r}}
$$

this gives that:

$$
\|u\|_{L^{q} H^{\alpha, r}} \lesssim \frac{\left\|u_{0}\right\|_{H^{\alpha}}}{1-\epsilon^{p-1}}
$$


Now, with Strichartz estimate:

$$
\begin{aligned}
\|u\|_{L \infty H^{\alpha}} & \lesssim\left\|u_{0}\right\|_{H^{\alpha}}+\left\|u|u|^{p-1}\right\|_{L^{q^{\prime}} H^{\alpha, r^{\prime}}} \\
& \lesssim\left\|u_{0}\right\|_{H^{\alpha}}+\epsilon^{p-1} \frac{\left\|u_{0}\right\|_{H^{\alpha}}}{1-\epsilon^{p-1}} \\
& \lesssim \frac{1+\epsilon^{p-1}}{1-\epsilon^{p-1}}\left\|u_{0}\right\|_{H^{\alpha}}
\end{aligned}
$$

Then for $\epsilon$ small we obtain

$$
\|u\|_{L_{T}^{\infty} H^{\alpha}}<\infty
$$

this gives that $T=\infty$.

\section{Now we are ready to prove Theorem 1.2 ,}

Let $u \in C\left([0, T] ; H_{r d}^{\alpha}\left(\mathbb{R}^{d}\right)\right.$ be the solution emanating from some initial datum $u_{0} \in H_{r d}^{\alpha}\left(\mathbb{R}^{d}\right)$. We have the following a priori estimates:

Lemma 3.1. Let $u \in C\left([0, T] ; H_{r d}^{\alpha}\left(\mathbb{R}^{d}\right)\right)$ be the solution of (1.1) emanating from $u_{0} \in H_{r d}^{\alpha}\left(\mathbb{R}^{d}\right)$. Then

$$
\|u\|_{L_{T}^{\infty} L^{2}} \leq\left\|u_{0}\right\|_{L^{2}} \text { and }\left\|(-\Delta)^{\frac{s}{2}} u\right\|_{L_{T}^{2} L^{2}} \leq \frac{1}{\sqrt{2 a}}\left\|u_{0}\right\|_{L^{2}} .
$$

Proof. Assume first that $u_{0} \in H^{\infty}\left(\mathbb{R}^{d}\right)$. Then (1.2) ensures that the mass is decreasing as soon as $u$ is not the null solution and (1.2) leads to

$$
\int_{0}^{T}\left\|(-\Delta)^{\frac{s}{2}} u(t)\right\|_{L^{2}}^{2} d t=-\frac{1}{2 a}\left(\|u(T)\|_{L^{2}}^{2}-\left\|u_{0}\right\|_{L^{2}}^{2}\right) \leq \frac{1}{2 a}\left\|u_{0}\right\|_{L^{2}}^{2} .
$$

This proves (3.1) for smooth solutions. The result for $u_{0} \in H^{\alpha}\left(\mathbb{R}^{d}\right)$ follows by approximating $u_{0}$ in $H^{\alpha}$ by a smooth sequence $\left(u_{0, n}\right) \subset H^{\infty}\left(\mathbb{R}^{d}\right)$.

From the first estimate in (3.1) we can obtain that:

$$
\|u\|_{L_{T}^{2} L^{2}} \leq T^{\frac{1}{2}}\|u\|_{L^{\infty} L^{2}} \leq T^{1 / 2}\left\|u_{0}\right\|_{L^{2}}
$$

and thus by interpolation:

$$
\left\|(-\Delta)^{\frac{\alpha}{2}} u\right\|_{L_{T}^{2} L^{2}} \lesssim\left\|(-\Delta)^{\frac{s}{2}} u\right\|_{L_{T}^{2} L^{2}}^{\frac{\alpha}{s}}\|u\|_{L_{T}^{2} L^{2}}^{1-\frac{\alpha}{s}} \lesssim \frac{1}{a^{\frac{\alpha}{s}}} T^{\frac{1}{2}\left(1-\frac{\alpha}{s}\right)}
$$

Interpolating now between (3.2) and the first estimate of (3.1) we get

$$
\|u\|_{L_{T}^{\theta} H^{\frac{2 \alpha}{\theta}}} \lesssim \frac{1}{a^{\frac{2 \alpha}{\theta s}}} T^{\frac{1}{2}\left(1-\frac{\alpha}{s}\right)} \text { where } \theta=\frac{4 \alpha}{d}+2
$$

and the embedding $H^{\frac{2}{\theta}}\left(\mathbb{R}^{d}\right) \hookrightarrow L^{\frac{4 \alpha}{d}+2}\left(\mathbb{R}^{d}\right)$ ensures that

$$
\|u\|_{L_{T}^{\theta} L^{\frac{4 \alpha}{d}+2}} \lesssim \frac{1}{a^{\frac{2 \alpha}{\theta s}}} T^{\frac{1}{2}\left(1-\frac{\alpha}{s}\right)} .
$$


Denoting by $T^{*}$ the maximal time of existence of $u$ and letting $T$ tends to $T^{*}$, this contradicts proposition (3.3) whenever $T^{*}$ is finite. This proves that the solutions are global in $H^{\alpha}\left(\mathbb{R}^{d}\right)$.

Remark 3.1. Note that for $s=\alpha$, we have that $\|u\|_{L_{T}^{\frac{4 \alpha}{d}+2}{ }_{L^{\frac{4 \alpha}{d}+2}} \lesssim 1 \text { for any }}$ $T>0$ which show that

$$
\|u\|_{L^{\frac{4 \alpha}{d}+2}\left(\mathbb{R}_{+}^{*} \times \mathbb{R}^{d}\right)}<+\infty
$$

In plus,

$$
\|u\|_{L^{\frac{4 \alpha}{d}+2}\left(\mathbb{R}_{+}^{*} \times \mathbb{R}^{d}\right)} \longrightarrow 0, \text { when } a \longrightarrow+\infty
$$

3.2. Proof Theorem 1.1. Now we will prove the global existence for small data, to do this we will use the following fractional Galgliardo-Niremberg inequalities (see [19]):

Lemma 3.2. Let $q, r$ be any real numbers satisfying $1 \leq q, r \leq \infty$, and $s$, $s_{1}$ be two reals numbers. If $u$ is any functions in $C_{0}^{\infty}\left(\mathbb{R}^{d}\right)$, then

$$
\left\|D^{s} u\right\|_{L^{p}} \leq C\left\|D^{s_{1}} u\right\|_{L^{r}}^{a}\|u\|_{L^{q}}^{1-a}
$$

where

$$
\frac{1}{p}=\frac{s}{d}+a\left(\frac{1}{r}-\frac{s_{1}}{d}\right)+(1-a) \frac{1}{q}
$$

for all $a$ in the interval

$$
\frac{s}{s_{1}} \leq a \leq 1
$$

where $C$ is a constant depending only on $d, s, s_{1}, q, r$ and $a$.

Now we have the following one:

Proposition 3.4. Let $\alpha>0$ and $u \in H^{\alpha}\left(\mathbb{R}^{d}\right)$. Then there exists $C=$ $C(d, \alpha)$ such that:

$$
\left\|(-\Delta)^{\frac{\alpha}{2}} u\right\|^{2}\left(\frac{1}{2}-C\|u\|_{L^{2}}^{\frac{4 \alpha}{d}}\right) \leq E(u(t))
$$

\section{Proof of proposition 3.4;}

We have that:

$$
E(u(t))=\frac{1}{2}\left\|(-\Delta)^{\frac{\alpha}{2}} u\right\|_{L^{2}}^{2}-\frac{d}{4 \alpha+2 d}\|u\|_{L^{\frac{4 \alpha}{d}+2}}^{\frac{4 \alpha}{d}+2}
$$

But by the fractional Gagliardo-Niremberq inequality there exists $A=$ $A(\alpha, d)$ such that:

$$
\int|u|^{\frac{4 \alpha}{d}+2} d x \leq A\left(\int\left|(-\Delta)^{\frac{\alpha}{2}} u\right|^{2} d x\right)\left(\int|u|^{2} d x\right)^{\frac{2 \alpha}{d}}
$$

then

$$
\begin{aligned}
E(u(t)) & \geq \frac{1}{2}\left\|(-\Delta)^{\frac{\alpha}{2}} u\right\|_{L^{2}}^{2}-A \frac{d}{4 \alpha+2 d}\left(\int\left|(-\Delta)^{\frac{\alpha}{2}} u\right|^{2} d x\right)\left(\int|u|^{2} d x\right)^{\frac{2 \alpha}{d}} \\
& =\left\|(-\Delta)^{\frac{\alpha}{2}} u\right\|_{L^{2}}^{2}\left(\frac{1}{2}-A \frac{d}{4 \alpha+2 d}\left(\int|u|^{2} d x\right)^{\frac{2 \alpha}{d}}\right) .
\end{aligned}
$$


Now let us return to the proof of theorem 1.1

Let $u \in C\left([0, T] ; H^{\infty}\left(\mathbb{R}^{d}\right)\right)$ be a solution to (1.1) emanating from $u_{0} \in$ $H^{\infty}\left(\mathbb{R}^{d}\right)$. Then it holds

$$
\frac{d}{d t} E(u(t))=-a \int\left|(-\Delta)^{\frac{s+\alpha}{2}} u(t)\right|^{2}+a \operatorname{Im} \int\left((-\Delta)^{s} u(t)\right)|u(t)|^{p-1} \overline{u(t)}
$$

and Hölder inequalities in physical space and in Fourier space lead to

$$
\left.\left|\int\left((-\Delta)^{s} u\right)\right| u\right|^{p+1} \mid \leq\left\|(-\Delta)^{s} u\right\|_{L^{2}}\|u\|_{L^{2 p+2}}^{p+1}
$$

with

$$
\left\|(-\Delta)^{s} u\right\|_{L^{2}} \leq\left\|(-\Delta)^{\frac{s+\alpha}{2}} u\right\|_{L^{2}}^{\frac{2 s}{s+\alpha}}\|u\|_{L^{2}}^{\frac{\alpha-s}{\alpha+s}} .
$$

Using Gagliardo-Nieremberg inequality, we obtain

$$
\|u\|_{L^{\frac{8 \alpha}{d}}+2}^{\frac{8 \alpha}{d}+2} \leq C_{d}^{\frac{8 \alpha}{d}+2}\|\nabla u\|_{L^{2}}^{4 \alpha}\|u\|_{L^{2}}^{\frac{8 \alpha}{d}+2-4 \alpha} .
$$

This estimate together with Cauchy-Schwarz inequality (in Fourier space)

$$
\|\nabla u\|_{L^{2}} \leq\left\|(-\Delta)^{\frac{s+\alpha}{2}} u\right\|_{L^{2}}^{\frac{1}{s+\alpha}}\|u\|_{L^{2}}^{\frac{(s+\alpha-1)}{s+\alpha}}
$$

lead to

$$
\|u\|_{L^{\frac{8 \alpha}{d}}+2}^{\frac{4 \alpha}{d}+1} \leq C_{d}^{\frac{4 \alpha}{d}+1}\left\|(-\Delta)^{\frac{s+\alpha}{2}} u\right\|_{L^{2}}^{\frac{2 \alpha}{s+1}}\|u\|_{L^{2}}^{\frac{2 s}{s+1}}\|u\|_{L^{2}}^{\frac{4 \alpha}{d}+1-\frac{2 \alpha}{s+\alpha}} .
$$

Combining the above estimates we eventually obtain

$$
\frac{d}{d t} E(u(t)) \leq a\left\|(-\Delta)^{\frac{s+\alpha}{2}} u\right\|_{L^{2}}^{2}\left(C_{d}^{\frac{4 \alpha}{d}+1}\|u\|_{L^{2}}^{4 \alpha / d}-1\right)
$$

which together with (3.1) implies that $E(u(t))$ is not increasing for $\left\|u_{0}\right\|_{L^{2}} \leq$ $\frac{1}{C_{d}^{1+\frac{d}{4}}}$ implies $E(u(t)) \leq E\left(u_{0}\right)$ for all $t \geq 0$. Now with proposition 3.4 in the hand we obtain that $\left\|(-\Delta u)^{\frac{\alpha}{2}}\right\|_{L^{2}} \lesssim E\left(u_{0}\right)$, for $\left\|u_{0}\right\|_{L^{2}}$ small enough. This finishes the proof.

Proof of theorem 1.3; The second part of this theorem was proved previously (see remark 3.1). Let us prove the scattering:

Let $v(t):=S_{-a,-\alpha,-s}(t) u(t)$ then

$$
v(t)=u_{0}+i \int_{0}^{t} S_{a, \alpha, s}(s)\left(|u|^{\frac{4 \alpha}{d}} u(s)\right) d s .
$$

Therefore for $0<t<\tau$,

$$
v(t)-v(\tau)=i \int_{\tau}^{t} S_{a, \alpha, s}\left(-t^{\prime}\right)\left(|u|^{\frac{4 \alpha}{d}} u\right) d t^{\prime}
$$

It follows from Strichartz's estimates (as previously) that:

$$
\|v(t)-v(\tau)\|_{L^{2}}=\left\|i \int_{\tau}^{t} S_{a, \alpha, s}\left(-t^{\prime}\right)\left(|u|^{\frac{4 \alpha}{d}} u\right)\right\|_{L^{2}} \leq\|u\|_{L^{\frac{4 \alpha}{d}+2}(t, \tau) L^{\frac{4 \alpha}{d}+2}}^{\frac{4 \alpha}{+}+1}
$$

But by remark (3.1) , for $s=\alpha$ we have that $u \in L^{\frac{4 \alpha}{d}+2}\left((0, \infty), L^{\frac{4 \alpha}{d}+2}\right)$, then the right hand side goes to zero when $t, \tau \longrightarrow+\infty$. The scattering follows from the Cauchy criterion. 


\section{REFERENCES}

[1] P. Antonelli and C. Sparber. Global well-posedness for cubic NLS with nonlinear damping. Comm. Partial Differential Equations, 35 (2010) 4832-4845.

[2] P. Antonelli, R. Carles and C. Sparber. On nonlinear Schrodinger-type equations with nonlinear damping. Int. Math. Res. Not. (2015), no. 3, 740-762.

[3] C. Bardos, P. Penel, P. Frisch and P.L. Sulem. Modified dissipativity for a nonlinear evolution equation arising in turbulence. Arch. Rat. Mech. Anal, 71 (1979) 4237-256.

[4] D.T. Blackstock. Generalized Burgers equation for plane waves. J. Acoust. Soc. Am. 77 (1985), no. 3, 2050-2053.

[5] T. Cazenave. Semilinear Schrödinger equations. volume 10 of Courant Lecture Notes in Mathematics. New York University Courant Institute of Mathematical Sciences, New York, 2003.

[6] T. Cazenave and F. Weissler, Some remarks on the nonlinear Schrödinger equation in the subcritical case, in New Methods and Results in Nonlinear Field Equations (Bielefeld, 1987), 59-69, Lecture Notes in Phys., 347, Springer, Berlin, 1989.

[7] T.Cazenave and F.Weissler. The Cauchy problem for the critical nonlinear Schrödinger equation. Nonlinear Anal. 14 (1990), 807-836.

[8] D. Cai, A. J. Majda, D.W.McLaughlin, and E.G.Tabak. Dispersive wave turbulence in one dimension. Phys. D 152/153 (2001), 551-572, Advances in nonlinear mathematics and science. MR 1837929 (2002k:76069)

[9] W. Chen and S. Holm. Fractional Laplacian time-space models for linear and nonlinear lossy media exhibiting arbitrary frequency power-law dependency. J. Acoust. Soc. Am. 115 (2004), no. 4, 1424-1430.

[10] Y. Cho, H. Hajaiej, G. Hwang, and T. Ozawa. On the Cauchy problem of fractional Schrödinger equation with Hartree type nonlinearity. Funkcial. Ekvac. 56 (2013), no. 2, 193-224. MR 3114821

[11] Y. Cho, G. Hwang, S. Kwon, and S.Lee. Profile decompositions and blowup phenomena of mass critical fractional Schrödinger equations. Nonlinear Anal. 86 (2013), 12-29. MR 3053553

[12] B. Dodson. Global well-posedness and scattering for the mass critical nonlinear Schrödinger equation with mass below the mass of the ground state. Advances in Mathematics 285 (2015), 1589-1618.

[13] L. Gaul. The influence of damping on waves and vibrations. Mech. Syst. Signal Process. 13 (1999), no. 1, 1-30.

[14] B. Guo, and D. Huang Existence and stability of standing waves for nonlinear fractional Schrödinger equations. J. Math. Phys. 53, 083702 (2012).

[15] B. Guo and Z. Huo.Global well-posedness for the fractional nonlinear Schrödinger equation. Commun. Partial Differential Equations 36(2), 247-255 (2010).

[16] Z. Guo, Y. Sire, Y. Wang, and L. Zhao.On the energy-critical fractional Schrödinger equation in the radial case. e-print arXiv:1310.6816 1 [math.AP] (2013).

[17] Z.Guo and Y. Wang. Improved Strichartz estimates for a class of dispersive equations in the radial case and their applications to nonlinear Schrdinger and wave equations. J. Anal. Math. 124(1), 1-38 (2014).

[18] A. Hanyga. Multi-dimensional solutions of space-fractional diffusion equations. Proc. R. Soc. London, Ser. A 457 (2001), 2993-3005.

[19] H.Hajaiej, L. Molinet, T.Ozawa and B. Wang. Necessary and sufficient conditions for the fractional Gagliardo-Nirenberg inequalities and applications to Navier-Stokes and generalized boson equations. RIMS Kokyuroku Bessatsu B26, 159-199 (2011).

[20] A. D. Ionescu and F.Pusateri.Nonlinear fractional Schrödinger equations in one dimension. J. Funct. Anal. 266 (2014), no. 1, 139-176. MR 3121725129. MR 3054599

[21] C.Klein, C.Sparber, and P.Markowich.Numerical study of fractional nonlinear Schrödinger equations. Proc. R. Soc. Lond. Ser. A Math. Phys. Eng. Sci. 470 (2014), no. 2172, 20140364, 26. MR 3269035

[22] C. E. Kenig, G. Ponce, and L. Vega.Well-posedness and scattering results for the generalized Korteweg-de-Vries equation via the contraction principle. Comm. Pure App. Math, 46 (1993), no. 4, 527-620. 
[23] J. Krieger, E. Lenzmann, and P. Raphael.Nondispersive solutions to the $L^{2}$-critical half-wave equation. Arch. Ration. Mech. Anal. 209 (2013), no. 1, 61-129.

[24] N. Laskin.Fractional Quantum Mechanics and Levy Path Integrals. Physics Letters 268 A 2000, 298-304.

[25] N. Laskin.Fractional Schrodinger equation. Physical Review E66: 0561082002.

[26] C. Miao, B. Yuan and B. Zhang. Well-posedness of the Cauchy problem for the fractional power dissipative equations. Nonlinear Analysis, 68 (2008) 461-484

[27] D. Stroock. Diffusion processes associated with Lévy generators. Z. Wahrscheinlichkeitstheorie und Verw. Gebiete 32 (1975), no. 3, 209-244.

[28] T. Saanouni. Remarks on damped fractional Schrödinger equation with pure power nonlinearity. Journal of Mathematical Physics 56, 061502 (2015); doi: $10.1063 / 1.4922114$

[29] M.I. Weinstein. Existence and dynamic stability of solitary wave solutions of equations arising in long wave propagation. Comm. Partial Differential Equations 12 (1987), no. 10, 1133- 1173 .

Mohamad Darwich: Faculty of Sciences, Laboratory of Mathematics, Doctoral School of Sciences and Technology, Lebanese University Hadat, Lebanon. 